\title{
Erratum to: Psychiatric comorbidities and photophobia in patients with migraine
}

\author{
Stefan Seidel", Roland Beisteiner, Maike Manecke, Tuna Stefan Aslan and Christian Wöber
}

\section{Erratum}

The original publication [1] contains an error in the 2nd paragraph of the introduction and one error in legend of Table 1.

The sentence should be: "These specific characteristics may significantly contribute to the psychosocial impairment and altered quality of life of these subjects."

The correct table legend can be found below: "DASS Depression Anxiety Stress Scale, N/A not applicable, NRS numeric rating scale, SD standard deviation. + Mann-Whitney-U-test; " chi square test".

Received: 17 February 2017 Accepted: 17 February 2017

Published online: 13 March 2017

\section{Reference}

1. Psychiatric comorbidities and photophobia in patients with migraine.

The Journal of Headache and Pain 2017 18:18 DOl:10.1186/s10194-017-0718-1

* Correspondence: Stefan.seidel@meduniwien.ac.at

Department of Neurology, Medical University of Vienna, Währinger Gürtel

18-20, A-1090 Vienna, Austria 